\title{
Prolongation structure of the KdV equation in the bilinear form of Hirota
}

\author{
G H M Roelofs and R Martini \\ Twente University of Technology, Department of Applied Mathematics, PO Box 217, \\ 7500 AE Enschede, The Netherlands
}

Received 27 October 1989, in final form 6 February 1990

\begin{abstract}
The prolongation strueture of the KdV equation in the bilinear form of Hirota is determined, the resulting Lie algebra is realised and the Bäcklund transformation obtained from the prolongation structure is derived. The results are compared with those found by Wahlquist and Estabrook and by Hirota.
\end{abstract}

\section{Introduction}

In their well known article [1] Wahlquist and Estabrook developed a prolongation technique for nonlinear evolution equations and applied it to the Korteweg-de Vries (KdV) equation. This resulted in a Lie algebra structure, which has been obtained independently by Estabrook [2] and van Eck [3], the latter merely using the algebraic foundations of this Lie algebra.

In [4] Hirota shows how nonlinear evolution equations may be transformed into bilinear differential equations. For this purpose it is convenient to write the $\mathrm{KdV}$ equation in potential form

$$
4 u_{t}=u_{x x x}+6 u_{x}^{2} .
$$

From equation (1) we obtain the bilinear equation

$$
\left(D_{1}^{4}-4 D_{1} D_{3}\right) \tau \cdot \tau=0
$$

where

$$
\left(D_{1}^{4}-4 D_{1} D_{3}\right) \tau \cdot \tau=\left.\left(\frac{\partial^{4}}{\partial y^{4}}-4 \frac{\partial}{\partial y} \frac{\partial}{\partial t^{\prime}}\right) \tau\left(x+y, t+t^{\prime}\right) \tau\left(x-y, t-t^{\prime}\right)\right|_{y=0, t^{\prime}=0}
$$

by putting

$$
u=\frac{\partial}{\partial x} \log \tau(x, t) .
$$

In this paper we will investigate the prolongation structure of the KdV equation in Hirota's bilinear form (2). Equation (2) is easily seen to admit an additional symmetry with group action $(x, t, \tau) \mapsto(x, t, \lambda \tau)$ which has no counterpart for the ordinary KdV. 
This investigation is motivated by the conjecture that this additional symmetry might be reflected in the prolongation structure.

Moreover, equation (2) is directly connected to the $\tau$-function approach of nonlinear evolution equations [5]. In a certain sense these $\tau$ functions are more primitive than the corresponding functions $u$, namely as an element of a group orbit $\tau$ stands closer to the underlying symmetry group than $u$ itself. This might also be reflected in the prolongation structure.

In section 2 we carry out the actual prolongation, after which in section 3 we obtain the resulting Lie algebra, in the sense that we give a faithful representation of it. Finally, in section 4 we derive the Bäcklund transformation obtained from the prolongation structure, which appears to be equal to the one found by Hirota.

\section{Prolongation}

Writing out equation (2) yields

$$
\tau \tau_{x x x x}-4 \tau_{x} \tau_{x x x}+3 \tau_{x x}^{2}-4 \tau \tau_{x t}+4 \tau_{x} \tau_{t}=0
$$

In order to express equation (4) by means of an ideal of differential forms we introduce

$$
p \equiv \tau_{x} \quad q \equiv p_{x}=\tau_{x x} \quad r \equiv q_{x}=\tau_{x x x}
$$

whereupon equation (4) may be written as the first-order equation

$$
\tau r_{x}-4 p r+3 q^{2}-4 \tau p_{t}+4 \tau_{t} p=0
$$

The set of first-order equations (5) and (6) may now be expressed by the following set of differential 2-forms

$$
\begin{aligned}
& \alpha_{1}=\mathrm{d} \tau \wedge \mathrm{d} t-p \mathrm{~d} x \wedge \mathrm{d} t \\
& \alpha_{2}=\mathrm{d} p \wedge \mathrm{d} t-q \mathrm{~d} x \wedge \mathrm{d} t \\
& \alpha_{3}=\mathrm{d} q \wedge \mathrm{d} t-r \mathrm{~d} x \wedge \mathrm{d} t \\
& \alpha_{4}=\tau \mathrm{d} r \wedge \mathrm{d} t+4 \tau \mathrm{d} p \wedge \mathrm{d} x-4 p \mathrm{~d} \tau \wedge \mathrm{d} x+\left(3 q^{2}-4 p r\right) \mathrm{d} x \wedge \mathrm{d} t
\end{aligned}
$$

where a solution manifold of equations (5) and (6) satisfies the condition

$$
\alpha_{1}=\alpha_{2}=\alpha_{3}=\alpha_{4}=0
$$

and vice versa. In order to ensure integrability of this system Cartan's theory of differential forms [6] imposes the condition $\mathrm{d} I \subset I$ on $I=I\left(\alpha_{1}, \ldots, \alpha_{4}\right)$, the ideal generated by the $\alpha_{i}$ in the exterior algebra with basis $\{\mathrm{d} x, \mathrm{~d} t, \mathrm{~d} \tau, \mathrm{d} p, \mathrm{~d} q, \mathrm{~d} r\}$; this condition is easily seen to be satisfied.

The prolongation method of Wahlquist and Estabrook may be described as follows: add a Lie algebra valued prolongation form

$$
\omega=\mathrm{d} y+F(\tau, p, q, r) \mathrm{d} x+G(\tau, p, q, r) \mathrm{d} t
$$


to the original system of differential forms and demand $\omega$ satisfies the condition

$$
\mathrm{d} \omega+[G, F] \mathrm{d} x \wedge \mathrm{d} t \in I\left(\alpha_{1}, \ldots, \alpha_{4}\right) .
$$

Condition (8) gives rise to the following system of overdetermined differential equations

$$
\begin{aligned}
& \tau F_{\tau}+p F_{p}=0 \\
& F_{p}-4 G_{r}=0 \\
& F_{q}=0 \\
& F_{r}=0 \\
& \tau p G_{\tau}+\tau q G_{p}+\tau r G_{q}+\left(4 p r-3 q^{2}\right) G_{r}+\tau[G, F]=0 .
\end{aligned}
$$

Using the method of characteristics, one derives from equations (9), (11) and (12) that

$$
F=F(p / \tau)
$$

The remaining equations were solved using a computer package for computations in prolongation theory described in [7]. If we define

$$
P=p / \tau \quad Q=q / \tau \quad R=r / \tau
$$

we find that

$$
\begin{aligned}
& F=x_{1}+4 P x_{2}+4 P^{2} x_{3} \\
& G=\left(R+P Q-2 P^{3}\right) x_{2}+\left(2 P R-Q^{2}-P^{4}\right) x_{3}+x_{4}-2 P x_{5}-2 P^{2} x_{6}-\left(Q+P^{2}\right) x_{7}
\end{aligned}
$$

where we have introduced

$$
x_{7}=\left[x_{2}, x_{1}\right] \quad x_{6}=\left[x_{2}, x_{7}\right] \quad x_{5}=\frac{1}{2}\left[x_{1}, x_{7}\right] .
$$

In addition we find the commutation relations

$$
\begin{array}{lll}
{\left[x_{1}, x_{3}\right]=2 x_{2}} & {\left[x_{1}, x_{4}\right]=0} & {\left[x_{1}, x_{5}\right]=2\left[x_{2}, x_{4}\right]} \\
{\left[x_{2}, x_{3}\right]=x_{3}} & {\left[x_{2}, x_{6}\right]=x_{7}} & {\left[x_{3}, x_{4}\right]=3\left[x_{2}, x_{5}\right]+x_{5} .}
\end{array}
$$

In the next section we will realise the Lie algebra determined by relations (17) and (18). Remark. From definition (14) we see that

$$
P_{x}=Q-P^{2} \quad Q_{x}=R-P Q
$$

whereupon equation (6) may be written as follows

$$
R_{x}=4 P_{t}+3 P R-3 Q^{2} \text {. }
$$

It is easily checked that this set of equations leads to the same prolongation structure as derived for equations (5) and (6). We see from this that both the $\mathrm{KdV}$ in Hirota's bilinear form and the prolongation structure belonging to it, are really determined by $\tau_{x} / \tau=(\log \tau)_{x}=u$ and not by $\tau$ itself. In fact, as we will see in section 5 , the results found here are the same as for the KdV equation in potential form.

Equations (19) and (20), however, will be useful in section 4, where we derive the Bäcklund transformation belonging to the prolongation structure. 


\section{Realisation of the prolongation algebra}

In this section we will determine the structure of the prolongation algebra of the $\mathrm{KdV}$ in Hirota's bilinear form and give a realisation of it, using the theory of Kac-Moody algebras. Henceforth we will denote this prolongation algebra, i.e. the Lie algebra generated by the letters $x_{1}, \ldots, x_{7}$ and subjected to relations (17) and (18), by HIR. An important tool for our purpose is a gradation. The following proposition is immediate.

Proposition 1. HIR admits a $\mathbb{Z}$ gradation with $\operatorname{deg}\left(x_{1}\right)=1, \operatorname{deg}\left(x_{2}\right)=0, \operatorname{deg}\left(x_{3}\right)=-1$, $\operatorname{deg}\left(x_{4}\right)=3, \operatorname{deg}\left(x_{5}\right)=2, \operatorname{deg}\left(x_{6}\right)=1$ and $\operatorname{deg}\left(x_{7}\right)=1$ such that relations (17) and (18) are homogeneous.

Using the computer package already mentioned and introducing new letters $x_{8}, \ldots, x_{15}$ we have computed (part of) the commutator table of HIR, which can be found in table 1 and in principle may be checked by hand using the Jacobi identity.

Table 1. Commutator table of HIR.

\begin{tabular}{|c|c|c|c|c|c|c|c|c|c|c|}
\hline & $x_{1}$ & $x_{2}$ & $x_{3}$ & $x_{4}$ & $x_{5}$ & $x_{6}$ & $x_{7}$ & $x_{8}$ & $x_{9}$ & $x_{10}$ \\
\hline$x_{1}$ & 0 & $-x_{7}$ & $2 x_{2}$ & 0 & $2 x_{8}$ & 0 & $2 x_{5}$ & $x_{9}$ & $2 x_{11}$ & 0 \\
\hline$x_{2}$ & & 0 & $x_{3}$ & $x_{8}$ & 0 & $x_{7}$ & $x_{6}$ & $x_{10}$ & 0 & $x_{8}$ \\
\hline$x_{3}$ & & & 0 & $x_{5}$ & $-x_{7}-x_{6}$ & $-2 x_{2}$ & $2 x_{2}$ & $-x_{5}$ & $-2\left(x_{8}+x_{10}\right)$ & $x_{5}$ \\
\hline$x_{4}$ & & & & 0 & $x_{11}$ & 0 & $x_{9}$ & $x_{12}$ & $x_{13}$ & 0 \\
\hline$x_{5}$ & & & & & 0 & $-2 x_{8}$ & $-2 x_{10}$ & $x_{14}$ & 0 & $-x_{11}$ \\
\hline$x_{6}$ & & & & & & 0 & $2 x_{5}$ & $x_{9}$ & $2 x_{11}$ & 0 \\
\hline$x_{7}$ & & & & & & & 0 & 0 & $-2 x_{14}$ & $-x_{9}$ \\
\hline$x_{8}$ & & & & & & & & 0 & $x_{15}$ & $-x_{12}$ \\
\hline$x_{9}$ & & & & & & & & & 0 & $-x_{13}$ \\
\hline$x_{10}$ & & & & & & & & & & 0 \\
\hline
\end{tabular}

Using this table and the gradation of proposition 1 we find that HIR contains a subalgebra isomorphic to $A_{1}=\mathrm{sl}(2)$, with standard basis $\{e, f, h\}$, where

$$
e=\frac{1}{2}\left(x_{7}-x_{6}\right) \quad f=x_{3} \quad h=-2 x_{2}
$$

and standard relations

$$
[e, f]=h \quad[h, e]=2 e \quad[h, f]=-2 f .
$$

Moreover we see that HIR contains a two-dimensional Abelian subalgebra

$$
H_{\text {Hir }}=\left\langle x_{1}-x_{6}, x_{4}-x_{10}\right\rangle
$$

commuting with all generators of HIR, hence consisting of central elements.

We recall from van Eck [3] that the prolongation algebra of the ordinary KdV is isomorphic to $H_{\mathrm{KdV}} \times\left(\mathbb{C}[\lambda] \otimes A_{1}\right)$, the direct product of a five-dimensional Heisenberg algebra $H_{\mathrm{KdV}}$ and the tensor product $\mathbb{C}[\lambda] \otimes A_{1}$. HIR will appear to have a similar structure, namely $H_{\mathrm{Hir}} \times\left(\mathbb{C}[\lambda] \otimes A_{1}\right)$, with $H_{\mathrm{Hir}}$ and $A_{1}$ as defined in (21) and (22).

To prove this we will need some results from the theory of Kac-Moody algebras, in particular the defining relations and the realisation of the infinite-dimensional Lie 
algebra $A_{1}^{(1)}$, i.e. the derived algebra of the infinite-dimensional Lie algebra $A_{1}^{(1)}[8]$. We have the following.

Proposition $2\left(\mathrm{Kac}\right.$ ). (a) $A_{1}^{(1) \prime}$ is the Lie algebra with generators $e_{0}, f_{0}, h_{0}, e_{1}, f_{1}, h_{1}$ and defining relations $(i, j=0,1)$ :

$$
\begin{array}{lll}
{\left[h_{i}, h_{j}\right]=0} & {\left[e_{i}, f_{j}\right]=\delta_{i j} h_{i}} \\
{\left[h_{i}, e_{j}\right]=a_{i j} e_{j}} & {\left[h_{i}, f_{j}\right]=-a_{i j} f_{j}} & \\
\left(\operatorname{ad} e_{i}\right)^{1-a_{i j}} e_{j}=0 & \left(\operatorname{ad} f_{i}\right)^{1-a_{i j}} f_{j}=0 & (i \neq j)
\end{array}
$$

where $a_{i i}=2$ and $a_{i j}=-2(i \neq j)$.

(b) $A_{1}^{(\mathrm{t}) \prime}$ is isomorphic to the algebra $\left(\mathbb{C}\left[\lambda^{-1}, \lambda\right] \otimes A_{1}\right) \oplus \mathbb{C} c$ where the isomorphism is given by

$$
\begin{array}{lll}
e_{0} \mapsto \lambda \otimes f & f_{0} \mapsto \lambda^{-1} \otimes e & h_{0} \mapsto-1 \otimes h+c \\
e_{1} \mapsto 1 \otimes e & f_{1} \mapsto 1 \otimes f & h_{1} \mapsto 1 \otimes h
\end{array}
$$

and the commutator on $\left(\mathbb{C}\left[\hat{\lambda}^{-1}, \lambda\right] \otimes A_{1}\right) \oplus \mathbb{C} c$ by

$$
\left[\left(\lambda^{k_{1}} \otimes x\right) \oplus \lambda_{1} c,\left(\lambda^{k_{2}} \otimes y\right) \oplus \hat{\lambda}_{2} c\right]=\left(\lambda^{k_{1}+k_{2}} \otimes[x, y]\right) \oplus k_{1} \delta_{k_{1},-k_{2}}(x \mid y) c
$$

with (...) an invariant bilinear symmetric form on $A_{1}$ with

$$
(x \mid y)= \begin{cases}1 & \text { if }(x, y)=(e, f),(f, e) \\ 2 & \text { if }(x, y)=(h, h) \\ 0 & \text { otherwise }\end{cases}
$$

Equating to zero $f_{0}$ and $h_{0}$ yields the following.

Proposition 3. The Lie algebra with generators $e_{0}, e_{1}, f_{1}, h_{1}$ and defining relations

$$
\begin{array}{ll}
{\left[e_{1}, f_{1}\right]=h_{1}} & {\left[h_{1}, e_{0}\right]=-2 e_{0}} \\
{\left[h_{1}, e_{1}\right]=2 e_{1}} & {\left[h_{1}, f_{1}\right]=-2 f_{1}} \\
\left(\operatorname{ad} e_{1}\right)^{3} e_{0}=\left(\operatorname{ad} e_{0}\right)^{3} e_{1}=0 &
\end{array}
$$

is isomorphic to the algebra $\mathbb{C}[\lambda] \otimes A_{1}$ where the isomorphism is given by

$$
\begin{array}{ll}
e_{0} \mapsto \lambda \otimes f & e_{1} \mapsto 1 \otimes e \\
f_{1} \mapsto 1 \otimes f & h_{1} \mapsto 1 \otimes h .
\end{array}
$$

We are now in a position to give a realisation of HIR.

Theorem 1. HIR is isomorphic to the algebra $H_{\mathrm{Hir}} \times\left(\mathbb{C}[\lambda] \otimes A_{1}\right)$. 
Proof. Put $H_{\mathrm{Hir}}=\left\langle c_{1}, c_{2}\right\rangle$ and let $L\left(e_{0}, e_{1}, f_{1}, h_{1}, c_{1}, c_{2}\right)$ be the free Lie algebra on generators $e_{0}, e_{1}, f_{1}, h_{1}, c_{1}, c_{2}$ and consider the Lie algebra morphism $\phi: L\left(e_{0}, e_{1}, f_{1}, h_{1}, c_{1}, c_{2}\right) \rightarrow$ HIR given by (cf formulae (21) and (22))

$$
\begin{array}{lll}
e_{0} \mapsto-\frac{1}{2}\left(x_{7}+x_{6}\right) & e_{1} \mapsto \frac{1}{2}\left(x_{7}-x_{6}\right) & f_{1} \mapsto x_{3} \\
h_{1} \mapsto-2 x_{2} & c_{1} \mapsto x_{1}-x_{6} & c_{2} \mapsto x_{4}-x_{10}
\end{array}
$$

Using table 1 it is easy to prove that $\phi$ leaves invariant relations (23), hence there is a Lie algebra morphism $\phi^{\prime}: H_{\mathrm{Hir}} \times\left(\mathbb{C}[\lambda] \otimes A_{1}\right) \rightarrow$ HIR. On the other hand if we denote $\lambda^{k} \otimes x$ by $\lambda^{k} x$, the Lie algebra morphism $\psi: L\left(x_{1}, \ldots, x_{7}\right) \rightarrow H_{H i r} \times\left(\mathbb{C}[\lambda] \otimes A_{1}\right)$ given by

$$
\begin{array}{lll}
x_{1} \mapsto-(e+\lambda f)-c_{1} & x_{2} \mapsto-\frac{1}{2} h & x_{3} \mapsto f \\
x_{4} \mapsto-\left(\lambda e+\lambda^{2} f\right)-c_{2} & x_{5} \mapsto \lambda h & x_{6} \mapsto-(e+\lambda f) \\
x_{7} \mapsto e-\lambda f & &
\end{array}
$$

preserves the relations (17) and (18), hence there exists a Lie algebra morphism $\psi^{\prime}:$ HIR $\rightarrow H_{H i r} \times\left(\mathbb{C}[\hat{\lambda}] \otimes A_{1}\right) . \phi^{\prime}$ and $\psi^{\prime}$ are easily seen to be each other's inverse.

\section{Bäcklund transformation}

Bäcklund transformations can be obtained from prolongation structures by realising the prolongation algebra in a appropiate way and putting

$$
y_{x}=-F \quad y_{t}=-G \text {. }
$$

In this way new solutions for the differential equation for which the prolongation structure was computed may be constructed [1].

Using the nonlinear representation of $\operatorname{sl}(2)$

$$
e=-y^{2} \quad f=1 \quad h=2 y
$$

we find for $F$ and $G$

$F=-\lambda+(y-2 P)^{2}$

$G=-\lambda^{2}-\lambda\left(-y^{2}+4 y P-3 P^{2}-Q\right)-y^{2}\left(P^{2}-Q\right)-y\left(R+P Q-2 P^{3}\right)-P^{4}-Q^{2}+2 P R$

which leads to the equations

$y_{x}=\lambda-(y-2 P)^{2}$

$y_{t}=\hat{\lambda}^{2}+\lambda\left(-y^{2}+4 y P-3 P^{2}-Q\right)+y^{2}\left(P^{2}-Q\right)+y\left(R+P Q-2 P^{3}\right)+P^{4}+Q^{2}-2 P R$

The compatibility condition $y_{x t}=y_{t x}$ is satisfied if $P, Q$ and $R$ are solutions of the $\mathrm{KdV}$ in Hirota's bilinear form ((19) and (20)). Solving $P$ from equation (27) and substituting the result into equation (28) we find that $y$ has to satisfy

$$
y_{x x}^{2}=\frac{4}{3}\left(y_{x}-\lambda\right)\left(y_{x x x}+3 y_{x}^{2}-4 y_{t}\right)
$$


On the other hand we can take the realisation of $\mathrm{sl}(2)$

$$
e=v^{2} \quad f=-1 \quad h=2 v
$$

yielding

$v_{x}=-\lambda+(2 P+v)^{2}$

$v_{t}=-\lambda^{2}+\lambda\left(+v^{2}+4 v P+3 P^{2}+Q\right)-v^{2}\left(P^{2}-Q\right)+v\left(R+P Q-2 P^{3}\right)-P^{4}-Q^{2}+2 P R$.

In the same way as before we find that $v$ has to satisfy

$$
v_{x x}^{2}=\frac{4}{3}\left(v_{x}+\lambda\right)\left(v_{x x x}-3 v_{x}^{2}-4 v_{t}\right)
$$

Now a simple observation shows that $v=-y$ transforms equation (32) into equation (29). Differentiating with respect to $x$ gives $v_{x}=-y_{x}$ from which we conclude that

$$
\lambda-(y-2 P)^{2}=\lambda-(2 \tilde{P}+v)^{2}=\lambda-(y-2 \tilde{P})^{2}
$$

where $\tilde{P}$ is another solution of equations (19) and (20). From this we find that $\tilde{P}=P$ or $\tilde{P}=y-P$. One easily checks by substitution that $\tilde{P}=y-P$ indeed satisfies equations (19) and (20) iff $P$ does, hence we have found the auto-Bäcklund transformation

$$
\tilde{P}=y-P .
$$

Differentiating equation (33) with respect to $x$ and using equation (27) to eliminate $y_{x}$ we obtain the same Bäcklund transformation in a more familiar form

$$
(\tilde{P}+P)_{x}+(\tilde{P}-P)^{2}=\hat{\lambda}
$$

which is the same as the one found by Wahlquist and Estabrook [1] and by Hirota [4].

\section{Conclusions}

If we take a closer look at equation (20), which is equivalent to the equation (6) for $\tau$, and use equation (19) to eliminate $Q$ and $R$, we find that $P$ has to satisfy

$$
4 P_{t}=P_{x x x}+6 P_{x}^{2}
$$

which is the KdV equation in potential form (1). Now the potential form of the KdV equation has already been discussed from the prolongation point of view by Kaup [9]. In fact, the present equations (15) and (16) are the same as equation (5) in Kaup's paper, but in different notation. Also the realisation that (30) and (31) are the same as Kaup's equation (12). However, in Kaup's paper the explicit form of the prolongation algebra has not been determined.

Hence we have found in a natural context that from the prolongation point of view Hirota's formalism is in fact nothing more than a way to obtain results, which could have been obtained without applying Hirota's formalism. As a consequence of this, 
the additional symmetry of the KdV equation in Hirota's bilinear form does not lead to extra information obtained from the prolongation structure of this equation.

\section{References}

[1] Wahlquist H D and Estabrook F B 1975 J. Math. Phys. 16

[2] Estabrook F B 1989 Proc. NATO Adv. Study Inst. 'Partially Integrable Nonlinear Evolution Equations and their Physical Applications' (Deventer: Kluwer)

[3] van Eck H N 1983 Proc. Kon. Ned. Akad. Wetensch. A 86

[4] Hirota R 1980 Solitons (Berlin: Springer) pp 157-76

[5] Date E, Jimbo M, Kashiwara M and Miwa T 1983 Proc. RIMS Symp. of Non-linear Integrable Systems-Classical Theory and Quantum Theory (World Scientific: Singapore)

[6] Cartan E 1971 Les Systèmes Différentiels Extérieurs et leurs Applications Géometrique (Paris: Hermann)

[7] Gragert P K H 1981 Symbolic computations in prolongation theory PhD Thesis Twente University of Technology

[8] Kac V G 1983 Infinite Dimensional Lie algebras (Progress in Mathematics 44) (Boston: Birkhäuser)

[9] Kaup D J 1982 AIP Conf. Proc. Mathematical Method in Hydrodynamics and Integrability in Dynamical Systems' (New York: AIP) pp 193-209 